\title{
Global Interannual Trends and Amplitude Modulations of the Sea Surface Height Anomaly from the TOPEX/Jason-1 Altimeters
}

\author{
Paulo S. Polito and Olga T. Sato \\ Instituto Oceanográfico, Universidade de São Paulo, São Paulo, Brazil
}

(Manuscript received 28 February 2007, in final form 31 October 2007)

\begin{abstract}
This study uses the global Ocean Topography Experiment (TOPEX)/Jason-1 altimeters' time series to estimate the 13-yr trend in sea surface height anomaly. These trends are estimated at each grid point by two methods: one fits a straight line to the time series and the other is based on the difference between the average height between the two halves of the time series. In both cases the trend shows large regional variability, mostly where the intense western boundary currents turn. The authors hypothesize that the regional variability of the sea surface height trends leads to changes in the local geostrophic transport. This in turn affects the instability-related processes that generate mesoscale eddies and enhances the Rossby wave signals. This hypothesis is verified by estimates of the trend of the amplitude of the filtered sea surface height anomaly that contains the spectral bands associated with Rossby waves and mesoscale eddies. The authors found predominantly positive tendency in the amplitude of Rossby waves and eddies, which suggests that, on average, these events are becoming more energetic. In some regions, the variation in amplitude over $13 \mathrm{yr}$ is comparable to the standard deviation of the data and is statistically significant according to both methods employed in this study. It is plausible that in this case, the energy is transferred from the mean currents to the waves and eddies through barotropic and baroclinic instability processes that are more pronounced in the western boundary current extension regions. If these heat storage patterns and trends are confirmed on longer time series, then it will be justified to argue that the warming trend of the last century provides the energy that amplifies both Rossby waves and mesoscale eddies.
\end{abstract}

\section{Introduction}

The global time series of sea surface height (SSH) anomaly collected by the Ocean Topography Experiment (TOPEX) and Jason-1 altimeters is presently more than $13 \mathrm{yr}$ long. These quasi-global SSH anomaly measurements are dominated by the heat storage variability (Chambers et al. 1997; Polito et al. 2000; Sato and Rossby 2000; Sato and Polito 2008) and therefore establish the connection between satellite altimetry and climate change studies. Although too short for the analysis of decadal and interdecadal processes, statistically significant linear tendencies at interannual scales can be obtained from the altimeter data. Within the framework of the geostrophic theory, the observed regional variability of the linear SSH tendencies should have dynamical consequences.

Corresponding author address: Paulo S. Polito, Instituto Oceanográfico, Universidade de São Paulo, Praça do Oceanográfico, 191, 05508-120 São Paulo SP, Brazil.

E-mail: polito@io.usp.br
The sea level changes mostly because of variations in heat and salt content of the oceans, in glacier and ice caps, and in Greenland and Antarctic ice sheets. Independent results (Bindoff et al. 2007) based on satellite altimetry account for each of these major factors and reach a globally averaged trend of $3.1 \pm 0.7 \mathrm{~mm} \mathrm{yr}^{-1}$ between 1993 and 2003. The inclusion of tide gauge data obtained before 1993 results in a trend of $1.8 \pm 0.5$ $\mathrm{mm} \mathrm{yr}^{-1}$ between 1961 and 2003. Similar results were presented and thoroughly discussed in Church et al. (2001) and Cazenave and Nerem (2004) and provide a basis of comparison for the present estimates.

Regional variations in sea level change rate were observed in both hydrographic and satellite data (Cabanes et al. 2001). From a dynamical point of view, regional variability of the rate of sea level change leads to changes in the surface and isopycnal slopes. That, in turn, locally changes the geostrophic velocity field and its horizontal gradient. From a thermodynamical point of view, regional variability of the rate of sea level change suggests localized variations in the temperature and, to a lesser degree, in the salinity throughout the 
water column (Sato et al. 2000), and therefore, horizontal density gradients. Back to the dynamics, through the thermal wind equations these gradients translate into vertical shear of horizontal velocities. Thus, in both cases, changes in the horizontal velocity gradient involve changes in the basic elements that condition instability processes: velocity shear and stratification. Our scientific hypothesis is that the regional variability of the 13-yr trend of SSH should have an observable influence in higher-frequency bands because it influences the instability processes.

The global SSH anomaly record is, in most latitudes, dominated by basin-scale signals that include the seasonal and interannual cycles (e.g., El Niño/La Niña, North Atlantic Oscillation, and Indian Ocean dipole).

Signals characterized as long and short baroclinic Rossby waves (Chelton and Schlax 1996; Polito and Cornillon 1997; Cipollini et al. 1997; Polito and Liu 2003) are often the second most energetic components at low and midlatitudes, in addition to the equatorial Kelvin waves (Polito et al. 2001). Because of the dependence of the critical latitude (Gill 1982) on the wave frequency, the more poleward the latitude, the lower the Rossby wave frequency threshold imposed by the dynamics. As a result, relatively short-period ( $\sim 50$ days) Rossby waves are found only at low latitudes; annual Rossby waves predominate at midlatitudes; and interannual Rossby waves reach their critical latitude near the Polar Circle (Polito and Liu 2003). Consequently, at high latitudes most of the Rossby wave signal vanishes, while mesoscale eddies gain amplitude (Polito and Liu 2003, their Fig. 7). Furthermore, there are local SSH maxima over the regions where all major western boundary currents (WBCs) turn east, namely, the Kuroshio, Gulf Stream, Agulhas, and Brazil Currents. In addition, local maxima are observed near the Antarctic circumpolar current and at the low latitudes of the Pacific and Indian Oceans.

The first objective of this study is to quantify and analyze the local interannual trends of the SSH obtained from altimeter. The next objectives are to quantify the trends of the amplitude of the seasonal cycle, baroclinic Rossby and Kelvin waves, and finally, of the mesoscale eddies. These are treated as potential responses to the long-term changes in SSH.

\section{Altimeter data}

The observational basis of this study is the TOPEX and Jason-1 altimeter data distributed by the Jet Propulsion Laboratory (JPL) Physical Oceanography Distributed Active Archive Center (PODAAC). The along-track interpolated geophysical data record is used; all standard corrections are applied (Benada 1997).

The altimeter data have a sampling period of 9.9156 days and an equatorial track separation of $315 \mathrm{~km}$ in the zonal direction. The original altimetric data are interpolated (Smith and Wessel 1990) into a regular $1^{\circ} \times$ $1^{\circ} \times 9.9156$ days grid and are called $\eta_{o}(x, y, t)$. A time series of $\eta_{o}$ maps from January 1993 to May 2006 was assembled and reorganized into zonal-temporal (Hovmoller) diagrams of $\eta_{o}(x, t)$, one per degree of latitude. At each basin and latitude, $\eta_{o}(x, t)$ is bandpass filtered into several additive components using an improved version of the method used in Polito et al. (2000), discussed in Polito and Liu (2003), and briefly explained here in section $2 \mathrm{a}$.

The methodology applied in the analysis of $\eta_{o}$ is a sequence of two-dimensional finite-impulse response filters (FIR), similar to that described in Polito and Liu (2003). The $\eta_{o}$ is decomposed into

$$
\begin{gathered}
\eta_{o}=\eta_{t}+\eta_{24}+\eta_{12}+\eta_{6}+\eta_{3}+\eta_{1}+\cdots \\
\cdots+\eta_{K 6}+\eta_{K 3}+\eta_{K 1}+\eta_{E},
\end{gathered}
$$

where $\eta_{t}$ is the nonpropagating, basin-scale signal and is dominated by seasonality and interannual variability. In this case a two-dimensional symmetric Gaussian filter is used to capture temporal scales of one year or more and wavelengths comparable to the local basin width. Further, $\eta_{24}$ to $\eta_{1}$ are the westward-propagating Rossby waves with approximate periods of 24, 12, 6, 3, and 1.5 months. The equatorial Kelvin wave signals $\eta_{K 6}$ to $\eta_{K 1}$ are present only in the equatorial region and refer to Kelvin waves with approximate periods of 6, 3, and 1.5 months. The remaining nonpropagating signal is taken as representative of mesoscale eddies $\eta_{E}$. However, in addition to eddies, it includes all the mesoscale signals that do not propagate as the waves above mentioned and the instrument noise. Each 2D FIR filter convolves a filter matrix with the data matrix to produce a bandpass-filtered output. For both Kelvin and Rossby waves the filter matrices are Gaussian-tapered cosinusoidal surfaces. The slope of these surfaces is associated with the phase speed of the waves in the passband. Therefore, the filter is able to distinguish not only eastward (positive slope) from westward (negative slope) propagation but also different phase speeds.

The filters are applied at each basin and latitude independently, in a sequence of decreasing periods and wavelengths. For each band the filtered output is removed from the remaining signal before the next filter is applied. The passband of these filters is broad by design, allowing for substantial variability in period and wavelength within each band. The bandwidth is such 
that there is a small overlap of adjacent bands, ensuring a complete coverage of the spectrum. Furthermore, although the periods are fixed, the wavelengths are iteratively adjusted toward the local maximum in amplitude. The data are processed globally, with a consistent methodology for all bands.

\section{Results and discussion}

The original altimeter signal is decomposed according to Eq. (1). The nonpropagating, basin-scale signal $\eta_{t}$ is dominated by seasonality and interannual variability. The time series is approximately $14 \mathrm{yr}$ long and this period sets the upper limit on what we refer to as the 13-yr trend. The amplitude of the annual cycle varies. In a few grid locations this variation can be large enough to influence the $13-\mathrm{yr}$ trend estimate. To avoid this effect, the local $\eta_{t}$ time series is smoothed with a 1D low-pass filter that cuts off nearly all of the annual and higher frequencies. A straight line is least squares fit to this smoothed curve, and the angular coefficient is regarded as the 13-yr trend.

To be conservative, all $1^{\circ} \times 1^{\circ}$ regions where there is no statistically significant linear trend were excluded. Two statistical parameters were calculated: $R$, the correlation coefficient between the data and the adjusted line and $P_{r}$, the probability of obtaining this same correlation by random chance, given the same number of degrees of freedom. Therefore, the proposed criteria eliminate the local series whose variability is far from linear $(R \leq 0.3)$ and those whose linear trend can be easily obtained by chance $\left(P_{r} \geq 0.3\right)$.

The global results obtained from this method are shown in Fig. 1. The linear trends based on the unfiltered (or original) altimeter data are shown in Fig. 1a, top. The trends of the basin-scale interannual signal are shown in Fig. 1b, second from the top. These patterns, particularly those in Fig. 1b are consistent with Fig. 7 in Cazenave and Nerem (2004). The relatively small discrepancies are attributed to the fact that, in this case, the data were filtered and, to a lesser degree, that the length of our time series is significantly longer.

It is important to notice that Table 1 presents regional averages of the trends estimated only from the unmasked areas in Fig. 1. A previous analysis of spatial averages that included the masked areas in Fig. 1 yielded results closer to the literature. The trends in these masked areas are regarded as statistically insignificant. They are on average positive, but relatively small in absolute value and thus their exclusion increases the area averages. In this first approach we adopted a conservative, yet statistically robust, method. An alternative approach is discussed next.

According to Table 1, the global mean SSH has a positive trend, mostly related to the basin-scale signals. Any row-wise comparison of the values in Table 1 must take into account that each of these averages used a different set of locations, selected according to the statistical parameters $R$ and $P_{r}$. Part of the difference between the original and basin-scale trends is a consequence of the masking of unreliable data indicated by the white areas in Fig. 1. The original data are noisier than the (filtered) basin scale, and thus only the most pronounced trends pass the statistical test based on $R$ and $P_{r}$ criteria, therefore increasing the average trend of the original data with respect to the basin-scale trend.

The criteria based on $R$ and $P_{r}$ excluded a significant number of points. Thus, a second method to estimate the trends was devised. The time series was separated in two periods of $7 \mathrm{yr}$. Instead of using all the data points, two mean SSHA values were obtained, one for each period. The trend is simply the slope of the line that connects these two points. Amplitude trends were computed in a similar way. The trends are statistically significant if and only if the two 7-yr means are different. Only locations where they are different with $95 \%$ statistical confidence are analyzed. These trends are shown in Fig. 2 and their regional averages are listed in Table 2.

The relative difference between regional averages in each of the two tables is similar, but the absolute trends in Table 2 are generally smaller. This happens because of the inclusion of many locations with small trends previously excluded by the $R$ and $P_{r}$ criteria of the first method. This puts the results in Table 2 in better agreement with Cazenave and Nerem (2004), who used all possible trends.

\section{a. Original signal}

Tables 1 and 2 show the largest ocean-averaged positive trends for the original signal $\left(\eta_{o}\right)$ in the Indian Ocean, followed by the Atlantic and Pacific. This is taken as an indication that these trends obtained from the altimeter records are not dominated by the variability associated to the El Niño-La Niña events of the last $13 \mathrm{yr}$. If that was the case, the largest trends within the period considered in this study would be expected on the Pacific. Furthermore, in Fig. 1 the equatorial Pacific and Indian basins are mostly masked out, and in Fig. 2 these regions are mostly included into the global mean by the second method. Nevertheless, in both cases the ocean-averaged trends are of the same order because the number of statistically relevant trends in the equatorial region is relatively small.

The paucity of statistically reliable data in large portions of Fig. 1a is a problem partially resolved in Fig. 2a. 


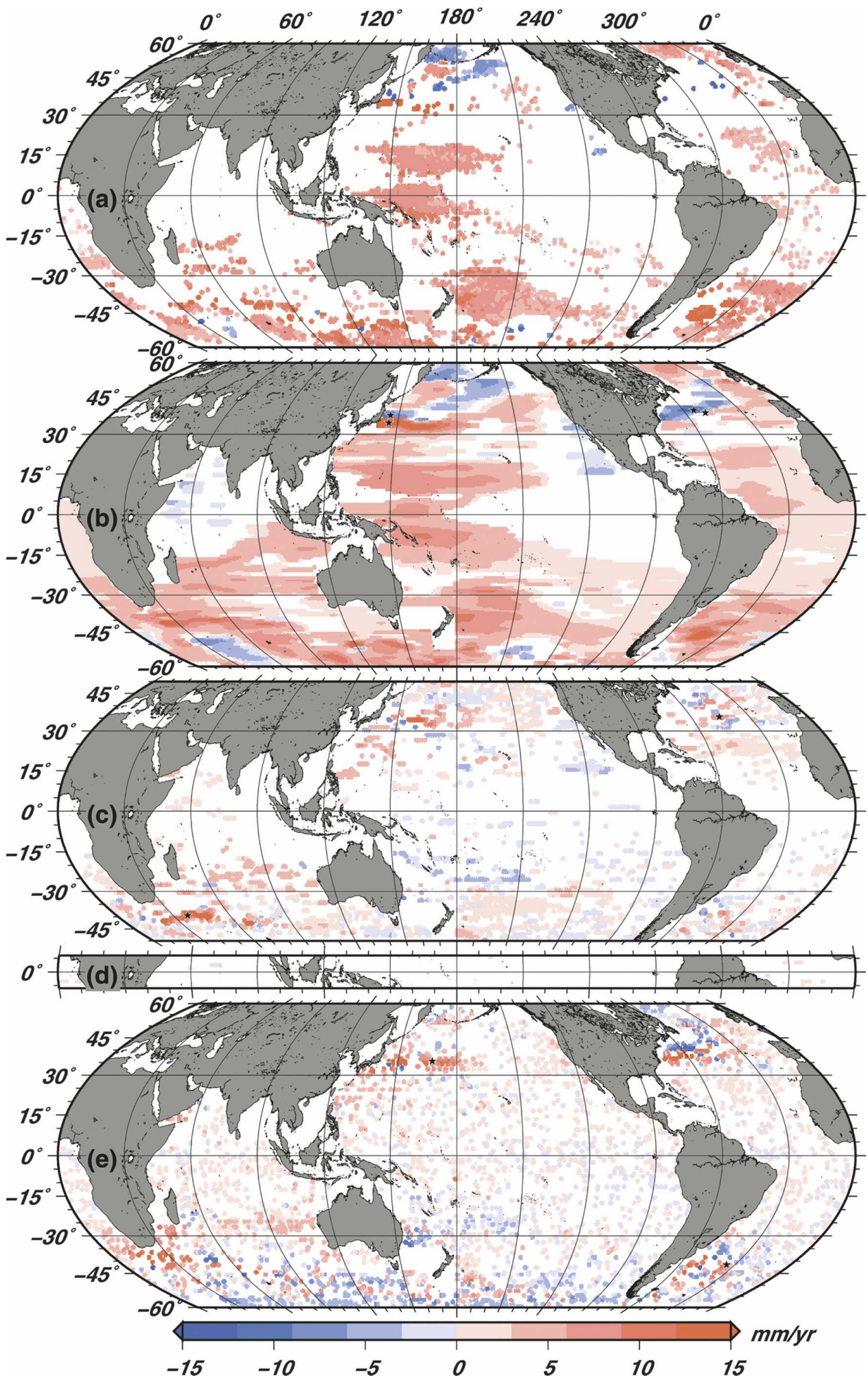

FIG. 1. Global maps of altimeter-derived SSH trends from the least squares fit method: (a) trend of the unfiltered altimeter data; (b) 13-yr trend of the basin-scale signal; (c) trend of the amplitude of the first-mode baroclinic Rossby waves; (d) trend of the amplitude of the equatorial Kelvin waves; (e) trend of the amplitude of the mesoscale eddies (all $\mathrm{mm} \mathrm{yr}^{-1}$ ). Areas shallower than $1000 \mathrm{~m}$ and small zonally enclosed regions are excluded because of the filtering process. Regions where $R \leq 0.3$ and $P_{r} \geq 0.3$ were excluded (white). The time series at the marked (black star) locations are seen in detail in Fig. 3 . 
TABLE 1. Global and regional averages of the trends based on least squares fit $\left(\mathrm{mm} \mathrm{yr}^{-1}\right)$ of the original altimeter data (Ori.), of the basin-scale nonpropagating signal (Bas.), and of the trends of the amplitude of the seasonal (Sea.), Rossby wave (Ros.), equatorial Kelvin wave (Kel.), and eddy signals (Edd.). These averages include only the locations whose trends present $R>0.3$ and $P_{r}<$ 0.3 . The regional average in the equatorial regions is taken over latitudes between $6.5^{\circ} \mathrm{S}$ and $6.5^{\circ} \mathrm{N}$; the northern (southern) regions are defined as north (south) of $6.5^{\circ} \mathrm{N}\left(6.5^{\circ} \mathrm{S}\right)$. In the North (South) Pacific, the eastern and western basins are divided by the $160^{\circ} \mathrm{W}\left(140^{\circ} \mathrm{W}\right)$ meridian; in the North (South) Atlantic, the eastern and western basins are divided by the $35^{\circ} \mathrm{W}\left(20^{\circ} \mathrm{W}\right)$ meridian; the North Indian basin was not divided, and in the South Indian Ocean the eastern and western basins are divided by the $90^{\circ} \mathrm{E}$ meridian.

\begin{tabular}{lrrrrrr}
\hline \multicolumn{1}{c}{ Region } & Ori. & \multicolumn{1}{c}{ Bas. } & \multicolumn{1}{c}{ Sea. } & Ros. & Kel. & Edd. \\
\hline Global & 6.30 & 3.65 & 0.12 & 1.34 & 0.09 & 0.50 \\
Pacific & 5.65 & 3.66 & 0.07 & 0.72 & - & 0.17 \\
Pacific N & 3.52 & 3.07 & 0.07 & 1.26 & - & 2.34 \\
Pacific NE & -0.19 & 1.60 & 0.29 & 0.31 & - & 1.39 \\
Pacific NW & 3.95 & 3.60 & -0.11 & 1.95 & - & 2.88 \\
Pacific equatorial & 7.34 & 5.56 & 0.92 & -1.24 & -0.85 & 0.58 \\
Pacific S & 6.49 & 3.81 & -0.16 & 0.40 & - & -1.31 \\
Pacific SE & 5.35 & 2.54 & -0.07 & 0.41 & - & -1.78 \\
Pacific SW & 6.85 & 4.86 & -0.24 & 0.39 & - & -0.89 \\
Atlantic & 6.50 & 3.13 & 0.01 & 1.15 & - & 0.78 \\
Atlantic N & 5.37 & 2.30 & -0.10 & 1.33 & - & 0.92 \\
Atlantic NE & 5.49 & 2.81 & -0.33 & 1.02 & - & 1.51 \\
Atlantic NW & 5.27 & 1.85 & 0.11 & 1.53 & - & 0.51 \\
Atlantic equatorial & 3.63 & 2.50 & 0.59 & - & 0.41 & 0.18 \\
Atlantic S & 7.21 & 3.78 & 0.04 & 0.94 & - & 0.76 \\
Atlantic SE & 5.98 & 3.07 & 0.51 & 0.96 & - & 0.98 \\
Atlantic SW & 8.73 & 4.68 & -0.42 & 0.92 & - & 0.53 \\
Indian & 7.92 & 4.30 & 0.31 & 2.99 & - & 0.87 \\
Indian N & - & -1.65 & -2.23 & 2.58 & - & 2.73 \\
Indian equatorial & 10.58 & 0.52 & 1.27 & 1.55 & -0.87 & 0.97 \\
Indian S & 7.91 & 4.50 & 0.34 & 3.07 & - & 0.79 \\
Indian SE & 8.49 & 4.95 & -0.15 & 1.39 & - & 0.15 \\
Indian SW & 7.25 & 4.14 & 0.66 & 4.26 & - & 1.27 \\
\hline & & & & & &
\end{tabular}

In both figures, the original signal shows larger average trends in all basins of the Southern Hemisphere, suggesting a possible warming or freshening of the South Atlantic Current (Sato and Polito 2008).

The western areas of the North and South Pacific and South Atlantic Oceans show significantly larger tendencies than their eastern counterparts. To a lesser degree, the opposite is observed in the South Indian and North Atlantic. In particular, the northwestern Atlantic trends are influenced by relatively large negative trends that occur in the eastern extension of the Gulf Stream.

\section{b. Basin-scale and seasonal signals}

There is a relatively large number of locations where the trends have no statistical significance. This is due to the influence of signals with smaller wavelength and higher frequencies. These signals are removed by the filters, and the trends based on the basin-scale SSH variability are statistically more robust. This is why the number of excluded areas in panel (b) of Figs. 1 and 2 is much smaller than that in panel (a). Therefore filtering is necessary, and the analysis focuses on the basinscale signals instead of on the original signals.

Most of the trends in the literature are estimated from smoothed time series and should be compared to panel (b) in Figs. 1 and 2. Inspection of $\eta_{t}$ on many locations revealed that the amplitude of the basin-scale SSH variability often changes significantly within a few degrees of latitude or longitude. Different trends in relatively close locations imply that there is a variation of the slope, and thus a trend in the strength of the geostrophic currents.

There are two locations in the Pacific, one at $34.5^{\circ} \mathrm{N}$, $145.5^{\circ} \mathrm{E}$ and the other at $37.5^{\circ} \mathrm{N}, 145.5^{\circ} \mathrm{E}$ marked with a star in Fig. 1. At both of these places the overall trend and $R\left(P_{r}\right)$ are particularly high (low). The trend is negative at $37.5^{\circ} \mathrm{N}$ and positive at $34.5^{\circ} \mathrm{N}$. This large change in SSH trend over a relatively short distance is not particular to the Kuroshio region. Similar results are clearly seen over the Gulf Stream and, in a less intense way, in all other WBCs. To be more precise, these extrema occur in the eastern extensions of the WBCs. The currents themselves are often partially located over regions less than $1000 \mathrm{~m}$ deep, and therefore outside the area resolved by the altimeter. This extreme case of the Kuroshio extension is shown in detail in the top two plots of Fig. 3, with two Gulf Stream locations depicted in the next two plots. It is important to notice from the extent of the vertical axis that the overall SSH amplitude is relatively large $(\sim 1 \mathrm{~m})$ in this region. The thin line shows $\eta_{o}$, and it is clear that its variability occurs over a wide spectral range, with a significant amount of energy in the high-frequency band. The FIR filtered basin-scale signal $\eta_{t}$ is shown in the slightly thicker line. In that, the seasonal signal is evident, particularly before 1997 . This is an indication that the spectral composition of the signal is variable. The seasonal signal, shown in a slightly thicker line, is obtained with a simple low-pass filter and removed. The remaining smooth signal (thicker line) is the basin-scale interannual residual variability, and its $13-y r$ trend is evident. The thickest straight line is the least squares fit to the smoothed signal. The title in the plots specifies the basin, the location, $R$, and $P_{r}$. For both currents statistically significant trends are observed with opposite signs at a short distance of each other.

The positive global average of the trend of the basinscale signal $\left(\eta_{t}\right)$ (Table 1$)$ is in close agreement with previous results (Cazenave and Nerem 2004). Similar to the original signal, the Indian Ocean presents the larg- 

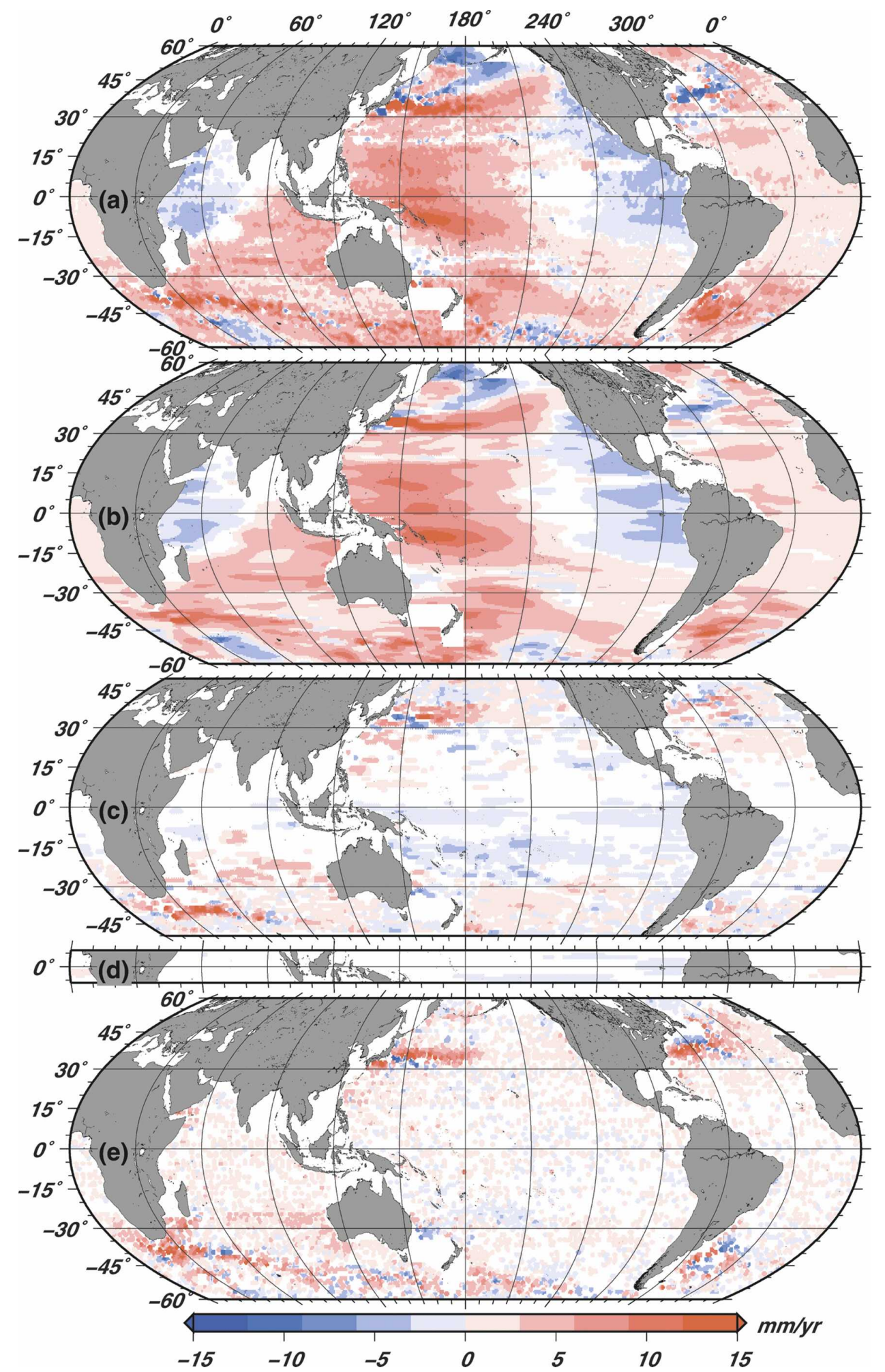

FIG. 2. Similar to Fig. 1 but for the trend obtained from the difference of two means taken over two periods, the first from 5 Jan 1993 to 28 Dec 1999 and the second from 14 Apr 1999 to 15 May 2006, divided by the time difference. Locations where the difference between the two means is below the $95 \%$ confidence level were excluded (white). 
TABle 2. Similar to Table 1. The first two columns show averages of the difference between two means: one taken from 5 Jan 1993 to 28 Dec 1999 and the other from 14 Apr 1999 to 15 May 2006, divided by the time difference; the last four columns show the difference between the amplitudes divided by $7 \mathrm{yr}$. These average trends $\left(\mathrm{mm} \mathrm{yr}^{-1}\right)$ include only the locations whose averages (or average amplitudes) are different with a confidence level above $95 \%$.

\begin{tabular}{lrrrrrr}
\hline \multicolumn{1}{c}{ Region } & Ori. & \multicolumn{1}{c}{ Bas. } & \multicolumn{1}{c}{ Sea. } & Ros. & Kel. & Edd. \\
\hline Global & 2.55 & 2.46 & 0.02 & 0.21 & -0.24 & 0.40 \\
Pacific & 2.34 & 2.35 & -0.18 & -0.09 & - & 0.22 \\
Pacific N & 2.05 & 2.11 & 0.01 & 0.37 & - & 0.59 \\
Pacific NE & 0.10 & 0.09 & 0.20 & -0.19 & - & 0.31 \\
Pacific NW & 3.36 & 3.47 & -0.11 & 0.75 & - & 0.79 \\
Pacific equatorial & 2.52 & 2.51 & -0.63 & -0.59 & -0.43 & 0.04 \\
Pacific S & 2.51 & 2.47 & -0.18 & -0.33 & - & 0.00 \\
Pacific SE & 1.01 & 0.90 & -0.04 & -0.12 & - & 0.00 \\
Pacific SW & 4.20 & 4.30 & -0.35 & -0.56 & - & 0.00 \\
Atlantic & 2.56 & 2.38 & 0.24 & 0.38 & - & 0.42 \\
Atlantic N & 1.84 & 1.70 & 0.21 & 0.57 & - & 0.67 \\
Atlantic NE & 2.19 & 2.18 & 0.10 & 0.51 & - & 0.76 \\
Atlantic NW & 1.57 & 1.33 & 0.30 & 0.61 & - & 0.61 \\
Atlantic equatorial & 1.95 & 2.09 & 0.52 & 0.01 & 0.28 & 0.03 \\
Atlantic S & 3.27 & 2.98 & 0.21 & 0.29 & - & 0.29 \\
Atlantic SE & 2.66 & 2.30 & 0.32 & 0.31 & - & 0.41 \\
Atlantic SW & 4.05 & 3.86 & 0.06 & 0.25 & - & 0.13 \\
Indian & 3.03 & 2.79 & 0.24 & 0.90 & - & 0.74 \\
Indian N & -1.70 & -1.58 & -0.98 & 0.40 & - & 0.53 \\
Indian equatorial & -0.66 & -0.75 & -0.44 & -0.56 & -0.22 & 0.49 \\
Indian S & 3.72 & 3.45 & 0.39 & 1.14 & - & 0.79 \\
Indian SE & 4.52 & 4.23 & 0.35 & 0.71 & - & 0.72 \\
Indian SW & 2.09 & 2.83 & 0.42 & 1.41 & - & 0.83 \\
\hline
\end{tabular}

est basin-scale trends and the Southern Hemisphere shows larger trends than the northern. The large trend over the equatorial Indian Ocean present in the $\eta_{o}$ analysis is no longer observed. This occurs because of the different selection masks, as seen in Figs. 1a and $1 \mathrm{~b}$. Furthermore, Fig. 2 complements these observations, as it reveals large unmasked areas, statistically significant according to the $95 \%$ confidence-level criterion.

The largest tendencies are observed in the Indian Ocean, followed by the Pacific and the Atlantic. Curiously, the only negative average results from the relatively small northern Indian Basin. The Southern Hemisphere shows, in the three basins (Tables 1 and 2), the largest tendencies. The western side of the Pacific and South Atlantic present larger tendencies than their eastern counterparts.

The trends in the equatorial regions are better portrayed by the results of the second method as it leaves a larger number of unmasked locations (Fig. 2 and Table 2). Trends are larger in the Pacific, followed by the Atlantic, with a small negative trend in the Indian basin.

To isolate the seasonal cycle, $\eta_{t}$ minus its smoothed interannual variability was used. A plot (not shown) of the tendency of the amplitude, similar to Fig. 1c, indicated that the amplitude of the annual cycle had, in general, a relatively small trend. Furthermore, these trends have predominantly low statistical significance; their $R\left(P_{r}\right)$ values are relatively low (high) compared with that of the Rossby waves or mesoscale eddies. For reference, the regional averages of the trend in amplitude of the seasonal cycle are shown in the fourth column of Tables 1 and 2.

\section{c. Rossby and Kelvin wave amplitudes}

To quantify a possible amplitude modulation of the Rossby wave signals, the sum of the FIR-filtered Rossby wave components $\left(\eta_{R}=\eta_{24}+\eta_{12}+\eta_{6}+\eta_{3}+\right.$ $\left.\eta_{1}\right)$ is calculated. This operation is performed in all grid points between the critical latitudes of the wave components. The absolute value of the $\eta_{R}$ time series was calculated and the local maxima estimated (Fig. 3). Through these local maxima a straight line was least squares fit. The trend is the angular coefficient of this line, $21 \mathrm{~mm} \mathrm{yr}^{-1}$ at $39.5^{\circ} \mathrm{S}, 37.5^{\circ} \mathrm{E}$ in the Indian Ocean and $10 \mathrm{~mm} \mathrm{yr}^{-1}$ at $35.5^{\circ} \mathrm{N}, 45.5^{\circ} \mathrm{W}$ in the Atlantic. These trends are local maxima with relatively large values compared to the SSH trend. Amplitude modulations are evident from the misalignment of the circles, yet these modulations are not perfectly described by a straight line as shown by the coefficients of determination $R$ of 0.58 and 0.55 , respectively. The tendency itself is significant as $P_{r}$ is, in both cases, much smaller than one. These results indicate a statistically reliable $13-\mathrm{yr}$ tendency to increase the amplitude of Rossby waves, in these cases mostly of annual and semiannual periods. Figure $1 \mathrm{c}$ shows the global distribution of this tendency for locations with $R>0.3$ and $P_{r}<0.3$. Similar to what was done for the SSH tendencies, the second method described above was used to estimate the trend in the amplitude of the oscillatory and transient signals. The trends obtained from the difference between two 7-yr averages are depicted in Figs. 2c-e and listed in Table 2.

In all but one region in Table 1, the mean trends of the amplitude of Rossby waves are positive, albeit less intense, less smooth, and statistically less robust than the 13-yr trend of the SSH. Furthermore, the latter is dominated by positive values (Fig. 1b), while the former (Fig. 1c) shows more of an equilibrium between positive and negative areas. Most of the region within $20^{\circ}$ of the equator is masked out due to low statistical significance. In this case, results from Table 2 differ from the previous results as small negative trends are observed all over the Pacific, except for the northwestern region. 


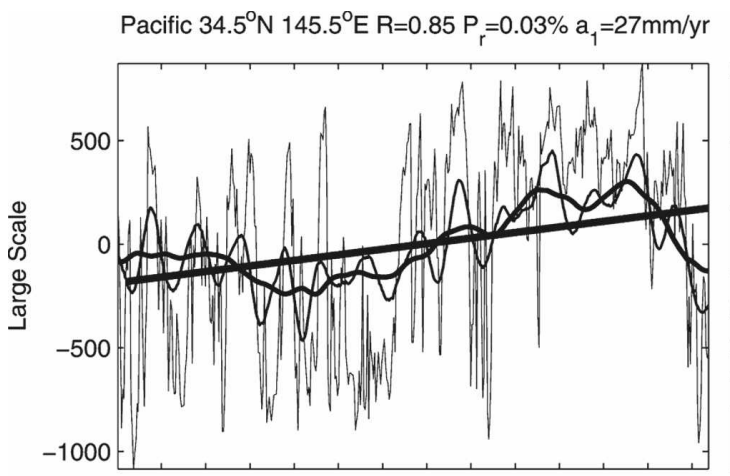

Pacific $37.5^{\circ} \mathrm{N} 145.5^{\circ} \mathrm{E} R=0.56 \mathrm{P}_{\mathrm{r}}=4.85 \% \mathrm{a}_{1}=-9 \mathrm{~mm} / \mathrm{yr}$
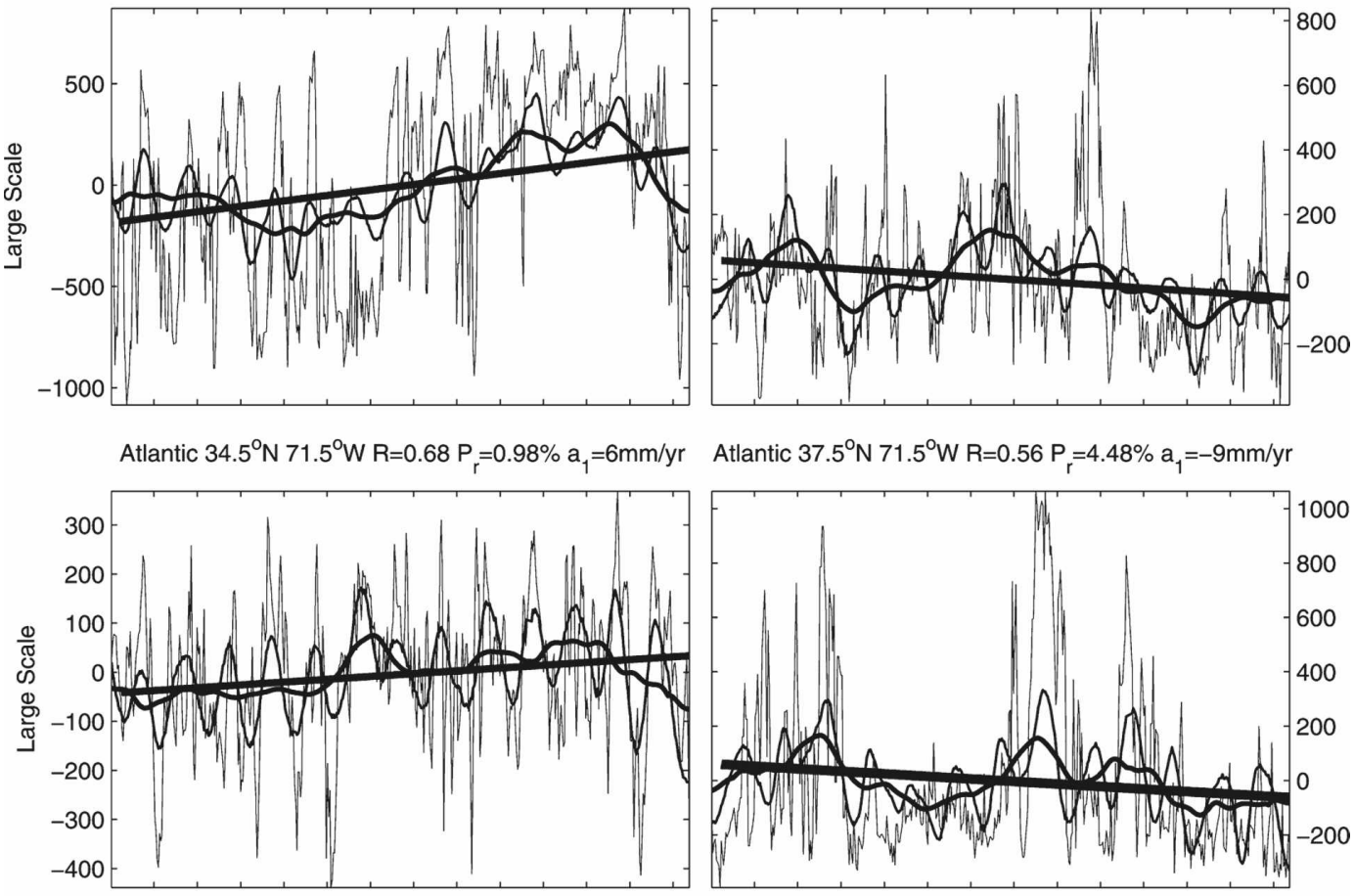

Atlantic $37.5^{\circ} \mathrm{N} 71.5^{\circ} \mathrm{W} R=0.56 \mathrm{P}_{\mathrm{r}}=4.48 \% \mathrm{a}_{1}=-9 \mathrm{~mm} / \mathrm{yr}$
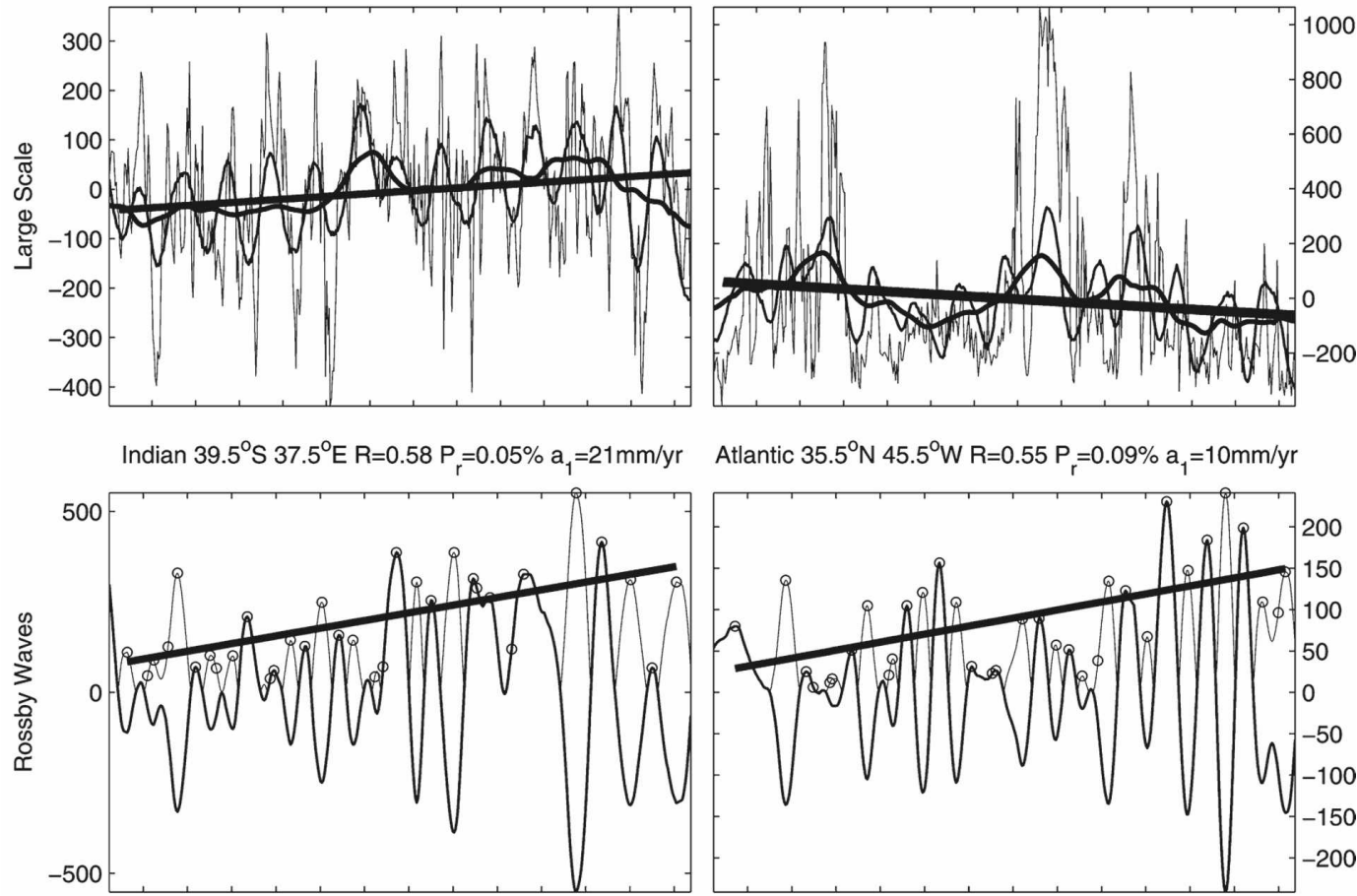

Atlantic $35.5^{\circ} \mathrm{N} 45.5^{\circ} \mathrm{W} R=0.55 \mathrm{P}_{\mathrm{r}}=0.09 \% \mathrm{a}_{1}=10 \mathrm{~mm} / \mathrm{yr}$

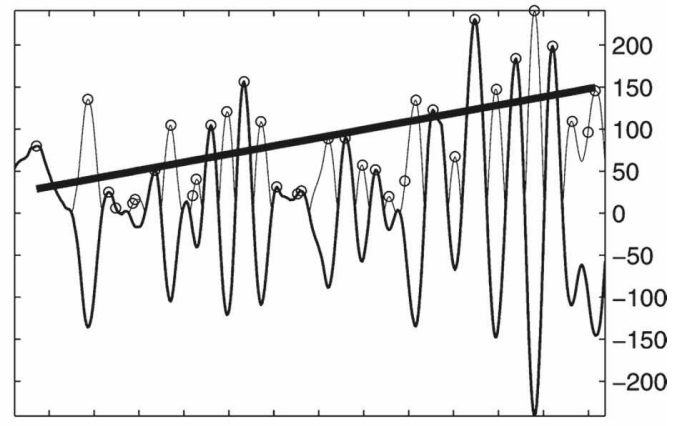

Pacific $35.5^{\circ} \mathrm{N} 167.5^{\circ} \mathrm{E} R=0.48 \mathrm{P}_{\mathrm{r}}=0.00 \% \mathrm{a}_{1}=10 \mathrm{~mm} / \mathrm{yr} \quad$ Atlantic $41.5^{\circ} \mathrm{S} 34.5^{\circ} \mathrm{W} R=0.47 \mathrm{P}_{\mathrm{r}}=0.00 \% \mathrm{a}_{1}=11 \mathrm{~mm} / \mathrm{yr}$
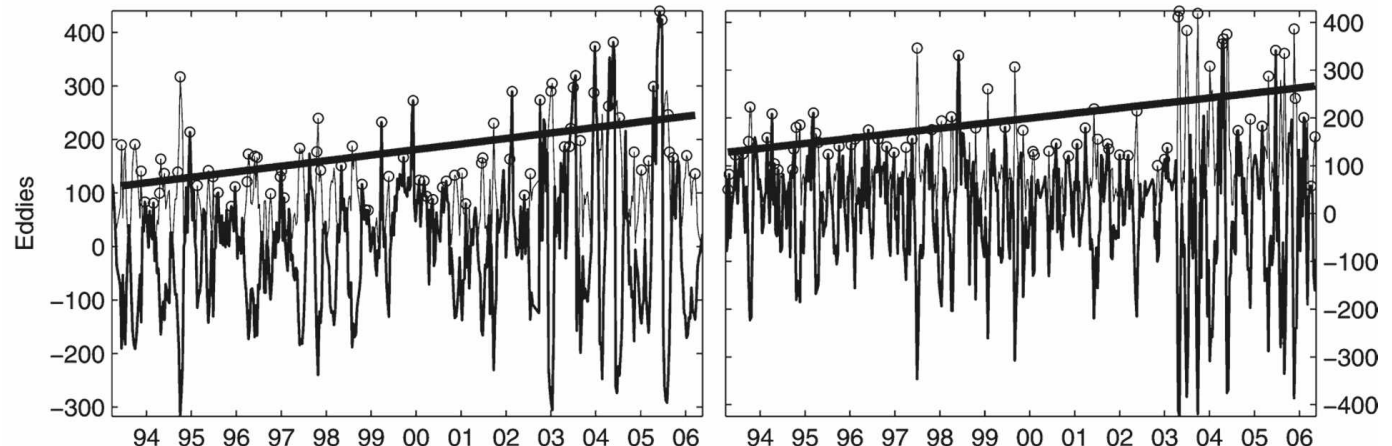

FIG. 3. These plots correspond to the marked locations in Fig. 1. Above each plot there is the basin name, location, coefficient of determination, probability of a random dataset to yield such coefficient, and the trend. The vertical axis shows the height anomaly $(\mathrm{mm})$ and the horizontal axis shows the time (yr). In the first row of plots the thinnest line shows the original altimetric time series, the second-most-thin line shows the nonpropagating, basin-scale signal $\eta_{t}$, the third shows the smoothed $\eta_{t}$ with the seasonal cycle removed, and the thickest line shows the linear trend. In the second row of plots the thick line shows the detrended annual cycle; the thin line shows its absolute value; the circles mark the local maxima; and the thick line shows the linear trend of the amplitude. Similarly, the third and fourth rows show results for the total Rossby wave signal and the mesoscale eddies. 
The Rossby wave amplitude trends are the largest in the Indian Ocean, followed by the Atlantic and Pacific, in consonance with the basin-scale trends: the waves grow larger where more energy becomes available. The southwestern Indian Ocean has the largest amplitude trend, which is even larger than its basin-scale trend in Table 1 . The peak trends in Rossby wave amplitude occur near western boundary current extensions where, through instability, the potential energy associated to heat storage becomes available.

Similar to the Rossby wave data processing, the equatorial Kelvin wave field is the sum of the eastwardpropagating signals obtained from the FIR filters $\left(\eta_{K}=\right.$ $\left.\eta_{K 6}+\eta_{K 3}+\eta_{k 1}\right)$. The global results for the trend in amplitude of equatorial Kelvin waves are shown in Figs. $1 \mathrm{~d}$ and $2 \mathrm{~d}$. In that, most of the area is masked out because of the lack of statistical robustness. The equatorial Pacific and Indian regions show a negative trend and the Atlantic shows a positive trend, and all are relatively small in both Tables 1 and 2 . In a global average, the amplitude tendency is approximately zero. The analysis is in part limited by the filter performance, which in this spectral band is not as good as in the Rossby waves case (Polito et al. 2000).

\section{d. Eddies}

The basin-scale nonpropagating signals, plus Rossby and Kelvin wave components, are subtracted from the original altimeter record. The residual contains mesoscale signals that do not propagate as equatorial Kelvin or Rossby waves. We assume that oceanic eddies are the dominant source of variability and thus refer to this spectral band simply as eddy signals. In addition, this spectral band includes the high-frequency part of the instrumental and geophysical noise, and possibly aliased tide residuals.

The tendency of the amplitude of the eddy signals is estimated through the same method as the previous amplitude trends. Figure 1e shows a map of the global distribution of the statistically robust amplitude trends. The largest trends are observed as nearly continuous patches that extend zonally in the vicinity of the eastward extension of the major WBCs and of the Antarctic Circumpolar Current. These regions show alternating patches of positive and negative amplitude trends. The marked locations, $35.5^{\circ} \mathrm{N}, 167.5^{\circ} \mathrm{E}$ over the Kuroshio Extension in the Pacific, and $41.5^{\circ} \mathrm{N}, 34.5^{\circ} \mathrm{W}$ over Gulf Stream extension in the Atlantic, are shown in detail in the last row of Fig. 3, and follow the same line convention as commented above. The coefficients of determination $R$ are not impressive: 0.48 and 0.47 , respectively. The probability $P_{r}$ that these trends occur out of ran- dom chance is nearly zero because the number of degrees of freedom is relatively large.

The global average of the trend of the amplitude of the eddy signal is positive and small, relative to the SSH trends. Except for the southern Pacific, all other regions present positive trends in Table 1. Table 2 shows no negative trends. The largest tendencies in the Pacific and Atlantic are obtained in the Northern Hemisphere. In particular, the northwest Pacific and southwest Indian regions present large trends, comparable to the regional average of the SSH trend in Table 1.

\section{Summary and conclusions}

The decomposition of the altimeter signal allowed for an advance in the interpretation of the variability of the satellite-based sea surface height record, which is now more than $13 \mathrm{yr}$ long. Two different trends are analyzed. The first is the trend of the sea surface height. In this study, different from Cazenave and Nerem (2004), the trend was calculated using both the original signal and the basin-scale, nonpropagating, nonseasonal part of the altimetric spectrum. The basin-scale, nonpropagating signal was obtained from a method based on 2D finite-impulse response filters (Polito et al. 2000). The main advantage of this method is that the transient signal due to Rossby waves, equatorial Kelvin waves, and mesoscale eddies is separated from the basin-scale signals that are most related to interannual variability. The seasonal signal was locally removed and a straight line was fit to this smoothed curve. The angular coefficient was taken as the 13-yr trend and is presented in Figs. 1 and 2. Area averages are presented under the label "Bas." in Tables 1 and 2.

To estimate the trend of the amplitude of the transient and oscillatory signals, the local maxima of the absolute value of the filtered SSHA are calculated. A straight line was least squares fit to these local maxima, and its angular coefficient represents the 13 -yr trend in amplitude. Mesoscale eddies, propagating Rossby and equatorial Kelvin waves, and seasonal signals were analyzed through this method. If, for example, the waves intensify, the trend will be positive; if the waves tend to vanish, the trend will be negative.

Locations where the trends are (i) far from linear ( $R \leq 0.3$ ) or (ii) the probability of obtaining the same trend by chance is large $\left(P_{r} \geq 0.3\right)$ were excluded from the analysis. At these locations the existence of a trend is not confirmed by our statistical analysis, and therefore it should not be included in the regional and global averages. Figure 1 shows the global distribution of the trends based on this linear least squares fit. Table 1 shows the regional averages obtained from this method. 
It is apparent, from the large masked-out areas, that this method may be too strict, as it excludes regions where the trends are robust but depart significantly from a straight line.

An alternative method to calculate the trends was devised, based on the difference of two means of the $\mathrm{SSH}$, calculated over two 7-yr periods. The regional averages were calculated only in the areas where the difference between the two means surpassed the $95 \%$ confidence level. Figure 2 shows the global map of the trends based on this method and Table 2 shows the corresponding regional averages.

Figures $1 \mathrm{~b}$ and $2 \mathrm{~b}$ show the $13-\mathrm{yr}$ trend of the basinscale, nonpropagating, nonseasonal signal. In general, it is very similar to the results in Cazenave and Nerem (2004). The trends are, in a global average, positive as expected from previous results. This positive trend is predominant over the Southern Hemisphere as indicated in Tables 1 and 2. This is an important result that conflicts with Intergovernmental Panel on Climate Change (IPCC) model predictions of larger average trends in the Northern Hemisphere (Church et al. 2001).

Local extrema in the 13-yr trend shown in Figs. 1b and $2 \mathrm{~b}$ occur over all major WBC eastward extensions. In these regions, maxima of opposite signs are often found within a few degrees of each other. This means that one side of the current has increased in height and the other side has decreased. An extreme case occurs in the Kuroshio region and is shown in detail in the top row of Fig. 3. The more plausible explanations for such a large change in slope are the intensification and northward shift in the path of the current, probably associated with a change in the large-scale wind stress curl (Seager et al. 2001; Qiu 2002; Ezer 1999; Frankignoul et al. 2002; Qiu and Chen 2005). The intensification of the horizontal shear makes these regions more prone to barotropic instabilities, and thus more intense variability is expected.

In spite of the relatively low spatial resolution of the altimeter data compared to the mean width of the current, the regions associated to positive and negative trends consistently spread over a large area. The FIR filters could possibly influence the zonal dimension, but not the meridional, because they are applied to $\eta_{o}(x, t)$ (Hovmoller diagrams), and each latitude is filtered independently of the others. Thus, given the continuous meridional extent of the positive and negative regions, they are very probably of geophysical origin.

The trend of the amplitude of the Rossby wave field is shown in Figs. 1c and 2c. The global mean trend is positive. In addition, significant positive trends are observed in the northwestern Pacific and southwestern
Indian Oceans. A large region with negative trends occupies the South Pacific. More intense positive trends are found in the western half of the basins, in midlatitudes and are shown in detail in Fig. 3, third row. In these cases, local maxima are not necessarily tied to the exact position of the western boundary currents, but spread over a larger surrounding area. The Rossby wave amplitude trends follow the basin-scale SSH trends, suggesting that the waves gain amplitude where more potential energy becomes available. This suggests that in these regions the mechanism that makes the energy available to the predominantly long, first-mode baroclinic Rossby waves is the baroclinic instability.

The trend of the amplitude in the spectral band identified as mesoscale eddies is shown in Figs. 1e and 2e. Although this spectral band may include noise from several sources, its analysis can still provide some selfconsistent information. The global average trend is positive and relatively small compared with the trend in Rossby wave amplitude. However, the North Pacific, the Indian Ocean, and the North Atlantic show, in average, positive trends. Negative trends are observed mostly in high latitudes of the Southern Hemisphere. In each basin, local maxima/minima occur exactly in the region where the most intense WBCs veer to the east. This is an indication of the intensification and possibly of a meridional shift in the mean position of the currents with their meanders and eddies. An argument similar to the one drawn for Rossby waves suggests that the intensification of mesoscale eddies is due to instabilities of the WBC system. In this case, the trends are larger and spread over a relatively small area. This is plausible as eddies are a much less efficient way to transfer energy than long Rossby waves.

Summarizing, this study has presented evidence that

- There are statistically significant $13-y r$ trends in the sea surface height, with a positive average in the Atlantic, Pacific, and Indian Oceans.

- These trends vary regionally; the spatial variability is more pronounced near the western boundary currents' eastward extension, suggesting an increase in the geostrophic velocity shear.

- On average, the amplitude of Rossby waves and mesoscale eddies is increasing in the vicinity of the western boundary currents' eastward extension.

This predominantly positive tendency in the amplitude of Rossby waves and eddies suggest that these transient events are, in a global average, becoming more energetic. In some regions, the variation in amplitude over $13 \mathrm{yr}$ is comparable to the standard deviation of the data and is statistically significant according to both methods employed in this study. The energy, in 
this case, is transferred from the mean flow to the waves and eddies through barotropic and baroclinic instability processes that are more pronounced in the WBC eastward extension regions. If these heat storage patterns and trends are confirmed on longer time series, then it will be justified to argue that the warming trend of the last century provides the energy that amplifies both Rossby waves and mesoscale eddies.

Acknowledgments. We thank the support from the Conselho Nacional de Desenvolvimento Cientifico e Tecnológico (CNPq) under Grant 477959/2004-0 and Fellowship 151722/2006-4 and from the Fundação para Amparo à Pesquisa do Estado de São Paulo (FAPESP) under Grant 2001/06921-3. We are grateful to the editor and to the anonymous reviewers for the excellent suggestions that contributed significantly to this manuscript.

\section{REFERENCES}

Benada, J. R., 1997: Merged GDR (TOPEX/POSEIDON) generation B MGDR-B version 2.0. Jet Propulsion Laboratory Physical Oceanography Distributed Active Archive Center (PO.DAAC) D-11007. [Available online at ftp://podaac. jpl.nasa.gov/data_collections/woce_v3/topex/docs/html/ usr_toc.htm.]

Bindoff, N., and Coauthors, 2007: Observations: Oceanic climate change and sea level. Climate Change 2007: The Physical Science Basis, S. Solomon et al., Eds., Cambridge University Press, 385-432.

Cabanes, C., A. Cazenave, and C. L. Provost, 2001: Sea level rise during past 40 years determined from satellite and in situ observations. Science, 294, 840-842.

Cazenave, A., and R. S. Nerem, 2004: Present-day sea level change: Observations and causes. Rev. Geophys., 42, RG3001, doi:10.1029/2003RG000139.

Chambers, D. P., B. D. Tapley, and R. H. Stewart, 1997: Longperiod ocean heat storage rates and basin-scale heat fluxes from TOPEX. J. Geophys. Res., 102, 10 525-10 533.

Chelton, D. B., and M. Schlax, 1996: Global observations of oceanic Rossby waves. Science, 272, 234-238.

Church, J., J. M. Gregory, P. Huybrechts, M. Kuhn, K. Lambeck, M. T. Nhuan, D. Qin, and P. L. Woodworth, 2001: Changes in sea level. Climate Change 2001: The Scientific Basis, J. T. Houghton et al., Eds., Cambridge University Press, 639-693.
Cipollini, P., D. Cromwell, M. S. Jones, G. D. Quartly, and P. G. Challenor, 1997: Concurrent altimeter and infrared observations of Rossby wave propagation near $34^{\circ} \mathrm{N}$ in the northeast Atlantic. Geophys. Res. Lett., 24, 889-892.

Ezer, T., 1999: Decadal variabilities of the upper layers of the subtropical North Atlantic: An ocean model study. J. Phys. Oceanogr., 29, 3111-3124.

Frankignoul, C., G. de Coëtlogon, T. M. Joyce, and S. Dong, 2001: Gulf Stream variability and ocean-atmosphere interactions. J. Phys. Oceanogr., 31, 3516-3529.

Gill, A. E., 1982: Atmosphere-Ocean Dynamics. International Geophysics Series, Vol. 30, Academic Press, 662 pp.

Polito, P. S., and P. Cornillon, 1997: Long baroclinic Rossby waves detected by TOPEX/POSEIDON. J. Geophys. Res., 102, 3215-3235.

— , and W. T. Liu, 2003: Global characterization of Rossby waves at several spectral bands. J. Geophys. Res., 108, 3018, doi:10.1029/2000JC000607.

_ O. T. Sato, and W. T. Liu, 2000: Characterization and validation of the heat storage variability from TOPEX/ POSEIDON at four oceanographic sites. J. Geophys. Res., 105, 16 911-16 921.

_- J. P. Ryan, W. T. Liu, and F. P. Chavez, 2001: Oceanic and atmospheric anomalies of tropical instability waves. Geophys. Res. Lett., 28, 2233-2236.

Qiu, B., 2002: The Kuroshio extension system: Its large-scale variability and role in the midlatitude ocean-atmosphere interaction. J. Oceanogr., 58, 57-75.

— , and S. Chen, 2005: Variability of the Kuroshio extension jet, recirculation gyre, and mesoscale eddies on decadal time scales. J. Phys. Oceanogr., 35, 2090-2103.

Sato, O. T., and T. Rossby, 2000: Seasonal and low-frequency variability in the meridional heat flux at $36^{\circ} \mathrm{N}$ in the North Atlantic. J. Phys. Oceanogr., 30, 606-621.

— nual heat storage trends in the Atlantic estimated from altimeters and Pilot Research Moored Array in the tropical Atlantic data. J. Geophys. Res., 113, C02008, doi:10.1029/ 2007JC004151.

,-- , and W. T. Liu, 2000: Importance of salinity measurements in the heat storage estimation from TOPEX/ POSEIDON. Geophys. Res. Lett., 27, 549-551.

Seager, R., Y. Kushnir, N. H. Naik, M. A. Cane, and J. Miller, 2001: Wind-driven shifts in the latitude of the KuroshioOyashio extension and generation of SST anomalies on decadal timescales. J. Climate, 14, 4249-4265.

Smith, W. H. F., and P. Wessel, 1990: Gridding with a continuous curvature surface in tension. Geophysics, 55, 293-305. 